\title{
Re: Safe and feasible inflow occlusion in laparoscopic liver resection
}

\author{
Giulio Belli · Corrado Fantini · Alberto D'Agostino • \\ Luigi Cioffi · Paolo Limongelli · Gianluca Russo · \\ Andrea Belli
}

Published online: 23 May 2009

(C) Springer Science+Business Media, LLC 2009

To the Editor:

We read with interest on the "online first" version of your journal the article by Cho et al. [1] reporting on safety and feasibility of a new technique of inflow occlusion in laparoscopic liver resection. The authors describe a method to laparoscopically encircle the hepatoduodenal ligament by vessel tape using an Endo Retract Maxi as a tourniquet for complete interruption of blood inflow to the liver (laparoscopic Pringle's manoeuvre). We agree with the authors' opinion on the ease and safety of hepatoduodenal ligament encircling, but would like to stress that this technique is not new.

In fact, to perform a laparoscopic Pringle's manoeuvre, we started to encircle the hepatoduodenal ligament by vessel tape using an Endo Retract Maxi several years ago.

We have already described this technique in the literature for the first time and stressed the feasibility and advantages of such a manoeuvre utilizing an Endo Retract Maxi [2].

\section{References}

1. Cho A, Yamamoto H, Nagata M, Takiguchi N, Shimada H, Kainuma $\mathrm{O}$ et al (2008) Safe and feasible inflow occlusion in laparoscopic liver resection. Surg Endosc. Dec 31

2. Belli G, Fantini C, D'Agostino A, Cioffi L, Limongelli P, Russo G et al (2008) Laparoscopic segment VI liver resection using a left lateral decubitus position: a personal modified technique. J Gastrointest Surg 12:2221-2226. Epub 2008 May 13
G. Belli $(\bowtie)$ - C. Fantini · A. D’Agostino - L. Cioffi ·

P. Limongelli · G. Russo - A. Belli

Department of General and Hepato-Pancreato-Biliary Surgery,

S.M. Loreto Nuovo Hospital, Via Cimarosa 2/A,

80127 Naples, Italy

e-mail: chirurgia.loretonuovo@tin.it

P. Limongelli

e-mail: limpao@libero.it 